\title{
Fatores de crescimento presentes no miocándio de pacientes com cardiopatia chagásica crônica
}

\author{
Growth factors in the myocardium of patients with \\ chronic chagasic cardiomyopathy
}

\author{
Marcia Martins Reis ${ }^{1}$, Maria de Lourdes Higuchi', \\ Vera Demarchi Aiello' e Luiz Alberto Benvenuti'
}

\begin{abstract}
Resumo Neste trabalho, quantificamos fatores de crescimento em fragmentos de miocárdio de 19 cardiopatas chagásicos crônicos com insuficiência cardíaca congestiva, através da técnica da imunoperoxidase. Pesquisamos: antígenos de T. cruzi, fatores de crescimento (GM-CSF, TGF- $\beta 1, P D G F-A$ e PDGF-B) e células inflamatórias $\left(C D 4^{+}, C D 8^{+}, C D 20^{+}\right.$e $\left.C D 68^{+}\right)$. A razão média $C D 4+/ C D 8+$ foi $0,6 \pm 0,3$. O número médio de macrófagos $\left(C D 68^{+}\right)$foi $5,9 \pm 3,1$; de células intersticiais PDGF- $A^{+}$foi $7,5 \pm 4,3 ; P D G F-B^{+} 2,9 \pm 2,7, T G F-\beta 1^{+} 2,2 \pm 1,9$ e GM-CSF+ 2,3 \pm 1,9. A marcação para PDGF-A foi geralmente intensa, ocorrendo também em endotélio, células musculares lisas e sarcolema; não houve correlação dessa positividade com a quantidade de células intersticiais positivas para os mesmos fatores. TGF- $\beta 1$ ocorreu em baixa expressão em $100 \%$ dos casos. Em conclusão, PDGF-A e B são, provavelmente, os fatores de crescimento mais relacionados às lesões proliferativas na cardiopatia chagásica crônica e, conseqüentemente, à fibrose. GM-CSF e TGF- $\beta 1$ estão pouco expressos. Não houve correlação estatisticamente significante entre os fatores de crescimento e a quantidade de parasita.
\end{abstract}

Palavras-chaves: Fatores de crescimento. Fibrose. Cardiopatia chagásica crônica humana. Doença de Chagas.

\begin{abstract}
In this work we quantified various growth factors in the myocardium of 19 patients with chronic chagasic cardiomyopathy and heart failure, through the immunoperoxidase technique. We looked for T. cruzi antigens, growth factors (GM-CSF, TGF- $\beta 1, P D G F-A$ and PDGF-B) and inflammatory cells $\left(C D 4^{+}, C D 8^{+}, C D 20^{+}\right.$and $\left.C D 68^{+}\right)$. The mean ratio of $C D 4+/ C D 8+T$ lymphocytes was $0.6 \pm 0.3$. The mean number of positive interstitial cells was $5.9 \pm 3.1$ for $C D 68^{+}$(macrophages); $7.5 \pm 4.3$ for $P D G F-A^{+} ; 2.9 \pm 2.7$ for $P D G F-B^{+}, 2.2 \pm 1.9$ for $T G F-\beta 1^{+}$and $2.3 \pm 1.9$ for $G M-C S F^{+}$. The immunoreaction for PDGF-A was intense, occurring also in the endothelium, smooth muscle cells and the sarcolemma; there was no correlation between the number of positive interstitial cells and the semiquantitation of the same growth factors in the other cells. TGF- $\beta 1$ presented low expression in 100\% of the cases. In conclusion, PDGF-A and B are probably the growth factors most related to the proliferative lesions and fibrosis present in chronic chagasic cardiomyopathy. GM-CSF and TGF- $\beta 1$ are present in low levels. There was no statistical correlation between growth factors and the quantity of the parasitic antigens.
\end{abstract}

Key-words: Growth factors. Fibrosis. Chronic human chagasic cardiopathy. Chagas' disease.

\footnotetext{
1 Instituto do Coração do Hospital das Clínicas da Faculdade de Medicina da Universidade de São Paulo, São Paulo, SP. Orgão financiador: FAPESP no. 1996/01075-7

Endereço para correspondência: Dra. Marcia Martins Reis. Lab. Anatomia Patológica/Instituto do Coração/USP, Av. Dr. Enéas de Carvalho Aguiar 44, subsolo, 05403-000 São Paulo, Brasil.

Tel: 5511 3069-5252; fax: 5511 3069-5251

e-mail: anpmarcia@incor.usp.br

Recebido para publicação em 7/12/99.
} 
A cardiopatia chagásica crônica (CCC) tem sido referida como um modelo para a cardiomiopatia dilatada humana em que, diferentemente da forma idiopática, a etiologia é conhecida. A dificuldade em se detectar o parasita no miocárdio e a falta de correlação entre sua presença e a intensidade da miocardite crônica levaram à postulação de teorias de auto-imunidade para explicar o desenvolvimento das lesões inflamatórias miocárdicas ${ }^{727}$. Entretanto, trabalhos recentes têm apontado, à semelhança do que foi descrito por Carlos Chagas ${ }^{6}$ e Vianna ${ }^{38}$, que o parasita tem participação ativa na patogenia da cardiopatia chagásica crônica ${ }^{3114}$.

Estudos com biópsia endomiocárdica mostram que a evolução clínica da doença de Chagas apresenta uma progressão contínua de destruição das fibras com fibrose e hipertrofia compensatória, com o aparecimento de dilatação e diminuição do desempenho ventricular ${ }^{21}$. A fibrose caracteriza-se por ser fina e difusa, envolvendo cada fibra cardíaca, com áreas de adensamento. A patogenia dessa fibrose ainda é desconhecida.

A presença de linfócitos e macrófagos nos infiltrados inflamatórios pode estar relacionada diretamente ao processo de fibrose, pela produção de citocinas e fatores de crescimento que provocariam a destruição tecidual e ativariam a fibrogênese ${ }^{823}$.

\section{MATERIAL E MÉTODOS}

Pacientes. Foram estudados 19 fragmentos transmurais de miocárdio da parede livre do ventrículo esquerdo, medindo cada um cerca de $2 \mathrm{~cm}$ de comprimento, de 19 pacientes com CCC e insuficiência cardíaca congestiva grave (grau III ou IV), sendo doze do sexo masculino e 7 do feminino, com idade média de 45 anos, com miocardite de grau discreto a intenso, e vieram a óbito no Instituto do Coração do Hospital das Clínicas da Faculdade de Medicina da Universidade de São Paulo. Os fragmentos foram obtidos até três horas pós-morte. O diagnóstico da doença de Chagas foi feito por laudos clínicolaboratoriais, com sorologia positiva para a doença. Este estudo foi aprovado pela Comissão Científica e de Ética do Instituto do Coração.

Reação de imunohistoquímica. Os fragmentos de miocárdio foram congelados em isopentano semilíquido e mantidos em nitrogênio líquido até 0 uso. De cada fragmento, foram feitos cortes seqüênciais em criostato (Reichert-Jung) a uma espessura de 4 micrômetros e fixados em
Células $T$ ativadas produzem linfocinas fibrogênicas tais como oTGF- $\beta$ (fator de crescimento transformante-beta) ${ }^{15}$. Os macrófagos secretam fatores que estimulam especificamente a proliferação de fibroblastos in vitro ${ }^{16}$ e aumentam a síntese de colágeno extracelular ${ }^{10}$.

A fibrose mais densa, em focos, na doença de Chagas, tem sido atribuída por muitos autores como conseqüência de fenômenos isquêmicos 12172036 .

Fatores de crescimento como TGF- $\beta 1$ e/ou PDGF (fator de crescimento derivado de plaquetas) também são citados em associação à disfunção respiratória na rejeição crônica de pulmão ${ }^{13}$, nas doenças renais crônicas ${ }^{5}$ e no infarto agudo do miocárdio ${ }^{35}$.

Tanto as áreas de fibrose maior (como na ponta do ventrículo esquerdo e parede basal inferior de ventrículo esquerdo), quanto a fina fibrose miocárdica intersticial presentes na CCC, que têm importante papel na insuficiência cardíaca desses pacientes, ainda é um assunto cuja patogenia não está esclarecida. O objetivo do nosso trabalho consiste na caracterização de alguns fatores de crescimento e como eles estão relacionados à presença de fibrose, se estão associados ou não a focos de inflamação e ao parasita, o que pode auxiliar no esclarecimento da patogenia da fibrose na doença de Chagas.

acetona anidra PA, durante 10 minutos à temperatura ambiente. Após a fixação, as lâminas foram estocadas em freezer $-80^{\circ} \mathrm{C}$ para posteriormente, serem submetidas à reação de imunoperoxidase, para estudo da presença de células ou estruturas positivas para: CD8, CD4, CD20, CD68, GM-CSF, TGF- $\beta 1$, PDGF-A, PDGF-B.

Após a hidratação com solução salina tamponada com fosfato (STF) pH 7,4, as secções foram tratadas com peróxido de hidrogênio a $0,3 \%$ em STF por 10 minutos, incubadas com soro fetal bovino não imune $10 \%$ em STF, por uma hora a $37^{\circ} \mathrm{C}$, e então incubados com os anticorpos primários diluídos em soro normal de porco (Dako, Glostrup, Denmark), 1,5\% em STF, por $18 \mathrm{~h}$, a $4^{\circ} \mathrm{C}$. Foram utilizados anticorpos monoclonais anti-humanos produzidos em camundongo, para identificar os subtipos de linfócitos $\mathrm{T} \mathrm{CD}^{+}, \mathrm{CD} 4^{+}$, linfócitos $\mathrm{B}\left(\mathrm{CD} 20^{+}\right)$, macrófagos $\left(\mathrm{CD}^{+} 8^{+}\right)$e policlonais anti-humanos produzidos em cabra, para fatores de crescimento 
PDGF-A, GM-CSF, e produzidos em coelho para TGF- $\beta 1$ e PDGF-B. As diluições de trabalho e a procedência dos anticorpos foram as seguintes: CD8 (1:10), CD4 (1:10), CD20 (1:1000) e CD68 (1:500), da Dako (Glostrup, Denmark); PDGF-B (1:20), da Genzyme (Cambridge, EUA); PDGF-A (1:100), GM-CSF (1:10) e TGFb1 (1:20), da Santa Cruz Biotechnology (Heildelberg, Germany). Como anticorpo de ligação foi utilizado antiimunoglobulina para as espécies cabra, camundongo ou coelho conjugada com a biotina, da Dako (Glostrup, Denmark), diluída a 1:200, por $1 \mathrm{~h}$, a $37^{\circ} \mathrm{C}$. Como reagente terciário foi utilizado estrepto-avidina conjugada com a peroxidase da Amersham International (Amersham, UK) diluída a 1:100, por $1 \mathrm{~h}$ a $37^{\circ} \mathrm{C}$.

Como controle positivo das reações de imunoperoxidase foram utilizados fragmentos de miocárdio de pacientes submetidos a transplante cardíaco e que não eram chagásicos, apresentando rejeição miocárdica aguda, além de secções de amígdala de pacientes submetidos a amigdalectomia. Como controle negativo, omitiu-se o anticorpo primário em secções dos casos estudados, para controle de possíveis ligações não específicas.

Para estudar a presença de antígenos de T. cruzi, lâminas com cortes histológicos de fragmentos de miocárdio das mesmas necropsias, fixados em formalina por $24 \mathrm{~h}$ e embebidos em parafina, com $4 \mu \mathrm{m}$ de espessura foram submetidas à reação de imunoperoxidase contra o $T$. cruzi. Controles positivos consistiram de cortes histológicos de miocárdio sabidamente com ninhos do parasita.

Após a desparafinação e bloqueio da peroxidase endógena com peróxido de hidrogênio a $3 \%$ em PBS por $15 \mathrm{~min}$, as lâminas foram submetidas à digestão proteolítica com uma solução de tripsina $0,015 \%$, da Dako (Glostrup, Denmark) e $\mathrm{CaCl}_{2}$ a $0,1 \%$ em $0,05 \mathrm{M}$ de TRIS/ $\mathrm{HCL} 0,015 \mathrm{M}$ de $\mathrm{NaCl}, \mathrm{pH} 7,8$, por $10 \mathrm{~min}$, a $37^{\circ} \mathrm{C}$. As secções foram incubadas com soro fetal bovino não imune $10 \%$ em STF, por $1 \mathrm{~h}$ a $37^{\circ} \mathrm{C} \mathrm{e}$ depois com soro imune anti-T. cruzi (produzido pelo Prof. Mortara, do Departamento de Parasitologia da Escola Paulista de Medicina da Universidade Federal de São Paulo), produzido em coelho, diluído 1:4.500 em BSA 1\% em STF, por $18 \mathrm{~h}$, a $4^{\circ} \mathrm{C}$. O anticorpo secundário usado foi anti-imunoglobulina de coelho produzido em porco conjugado com a biotina, da Dako (Glostrup, Denmark), diluído a 1:800 por 30min, a $37^{\circ} \mathrm{C}$. Como composto de ligação para a biotina foi utilizada estrepto-avidina conjugada com a peroxidase, da Amersham International (Amersham, UK), diluída a $1: 100$, por $30 \mathrm{~min}$, a $37^{\circ} \mathrm{C}$.

As lâminas submetidas a reações de imunoperoxidase foram colocadas por $10 \mathrm{~min}$ em uma solução contendo $40 \mathrm{mg}$ de 3,3 'diaminobenzidina da Sigma Chemical Corporation (St. Louis, MO) e $600 \mathrm{ml}$ de peróxido de hidrogênio a $6 \%$ em $100 \mathrm{ml}$ de PBS, $\mathrm{pH} 7.4$ e depois contracoradas com hematoxilina de Harris.

Análise da reação de imunohistoquímica. As células inflamatórias interstíciais positivas pela reação de imunoperoxidase foram identificadas pelo produto colorido marrom contornando a membrana celular no caso das reações para linfócitos CD4, CD8, CD20 (linfócito B) e no citoplasma das demais imunorreações. Foi feita a quantificação das respectivas células contandose o número de células positivas em 25 campos no aumento de 400x, em microscópio óptico, e calculada a média de células positivas por campo.

Para avaliação da presença de antígenos de T. cruzi no miocárdio, utilizou-se análise semiquantitativa, seguindo o seguinte critério: 0 , ausência de imunomarcação; 1 , presença de poucos antígenos do T. cruzi fora da fibra cardíaca (na matriz extracelular ou no citoplasma de macrófagos) e 2 , presença de pseudocisto no citoplasma de fibra cardíaca e de antígenos do T. cruzi fora da fibra cardíaca.

Foi feita também análise semiquantitativa para avaliação da intensidade de positividade da reação de imunohistoquímica em células endoteliais, células musculares lisas, sarcolema e matriz extracelular nas detecções de PDFG-A, PDGF-B, TGF- $\beta 1+e$ GM-CSF. A semiquantificação foi de 0 a 3 , de acordo com a intensidade cromogênica, sendo 0 , ausência de coloração; 1, discreta; 2, moderada e 3, intensa.

Adicionalmente, os corações foram pesados, e também, avaliada a intensidade de miocardite em cortes corados pela HE.

Análise estatística. Inicialmente, realizou-se análise descritiva das variáveis para resumir os dados através dos valores mínimo e máximo, mediana, média e desvio-padrão.

Para avaliar a relação entre as variáveis semiquantitativas estudadas, utilizou-se o coeficiente de correlação de Spearman ${ }^{25}$ e, para as variáveis quantitativas, o coeficiente de 
Pearson ${ }^{25}$. O teste de Wilcoxon ${ }^{24}$ foi empregado para comparação entre a intensidade de positividade de antígenos do $T$ cruzi ( 0 a 2) e as variáveis idade, peso do coração, número de células positivas para CD8, CD4, CD68, CD20, PDGF-A, PDGF-B, TGF- $\beta 1$ e GM-CSF.
Os resultados das análises foram interpretados utilizando-se o nível de significância de 5\%. No caso das relações entre medidas quantitativas, considerou-se importante um coeficiente de correlação ( $r$ ) maior que 0,70 . Os cálculos foram feitos por meio do sistema computacional SAS ${ }^{2829}$.

\section{RESULTADOS}

A Tabela 1 revela resultados individuais da análise quantitativa bem como as médias, desvios padrão, medianas, mínimos e máximos. Fornece, ainda, dados sobre a presença de antígenos do T. cruzi e outras características clínicas e anatomopatológicas de cada caso. As médias e desvios padrão dos números médios de células inflamatórias positivas/campo de 400x dos 19 casos estudados foram: $6,1( \pm 4,4)$ para células T CD4+; 10,9 ( $\pm 6,8)$ para células T CD8*; 1,3 $( \pm 1,0)$ para células B $\left(C D 20^{+}\right) ; 5,9( \pm 3,1)$ para macrófagos $\left(\mathrm{CD}^{+} 8^{+}\right) ; 7,5( \pm 4,2)$ para células PDGF-A; 2,9 ( $\pm 2,7)$ para células PDGF-B; 2,2 $( \pm 1,9)$ para células TGF- $\beta 1$ e $2,3( \pm 1,9)$ para células GM-CSF (Figura 1A-F, H).
A semiquantificação de antígenos do T. cruzi variou de 0 a 2 e os resultados estão na Tabela 1. Apenas um caso foi negativo sendo observada quantidade escassa (1) ou moderada (2) de antígenos do T.cruzi em 18 dos 19 casos estudados (Figura 1G).

O número médio de linfócitos $\mathrm{T} \mathrm{CD}^{+}$foi mais alto que o de linfócitos T CD4 ${ }^{+}$, com razão $\mathrm{CD}^{+} / \mathrm{CD}^{+}$0,6 $( \pm 0,3)$. O número médio de células $\mathrm{CD}^{+} 8^{+}$foi $5,9( \pm 3,1)$. O número de células PDGFA positivas foi muito alto $-7,5( \pm 4,3)$, comparado aos outros fatores de crescimento, em que os números médios de células positivas para PDGFB $(2,9 \pm 2,7)$, TGF- $\beta 1(2,2 \pm 1,9)$ e GM-CSF $(2,3 \pm 1,9)$ foram bem mais baixos (Tabela 1$)$.

Tabela 1 - Análise quantitativa das células inflamatórias e fatores de crescimento, dados clínicos e anatomopatológicos dos 19 pacientes com cardiopatia chagásica crônica.

\begin{tabular}{|c|c|c|c|c|c|c|c|c|c|c|c|c|c|c|c|}
\hline Caso & Paciente & Idade & Sexo & $\begin{array}{l}\text { Intensidade } \\
\text { inflamatória }\end{array}$ & $\begin{array}{l}\text { Antígenos } \\
\text { de } T \text {. cruzi }\end{array}$ & CD8 & CD4 & $\begin{array}{l}\text { CD4/ } \\
\text { CD8 }\end{array}$ & CD68 & CD20 & $\begin{array}{c}\text { PDGF } \\
\text { A }\end{array}$ & $\begin{array}{c}\text { PDGF } \\
\text { B }\end{array}$ & $\begin{array}{c}\text { TGF- } \\
\beta 1\end{array}$ & $\begin{array}{l}\text { GM- } \\
\text { CSF }\end{array}$ & $\begin{array}{l}\text { Peso } \\
\text { (g) }\end{array}$ \\
\hline 1 & OAF & 54 & M & moderada & 1 & 12,5 & 9,7 & 0,7 & 6,1 & 0,5 & 3,7 & 2,8 & 1,7 & 0,3 & 450 \\
\hline 2 & CMC & 25 & M & moderada & 2 & 14,2 & 4,4 & 0,3 & 9,8 & 0,9 & 6,5 & 1,9 & 1,5 & 5,1 & 500 \\
\hline 3 & FMP & 22 & $\mathrm{~F}$ & intensa & 2 & 7,6 & 3,9 & 0,5 & 9,2 & 1,5 & 4,2 & 1,0 & 0,3 & 3,0 & 500 \\
\hline 4 & EPE & 60 & $\mathrm{~F}$ & discreta & 2 & 17,6 & 6,0 & 0,3 & 3,5 & 3,4 & 9,5 & 1,8 & 1,0 & 0,9 & 500 \\
\hline 5 & LMO & 40 & $M$ & moderada & 0 & 14,2 & 10,3 & 0,7 & 12,3 & 2,9 & 9,6 & 2,7 & 4,9 & 2,2 & 700 \\
\hline 6 & JCP & 60 & $\mathrm{M}$ & moderada & 1 & 24,2 & 17,2 & 0,7 & 3,0 & 0,5 & 2,0 & 2,8 & 1,5 & 0,6 & 420 \\
\hline 7 & SCL & 30 & M & moderada & 1 & 22,8 & 13,5 & 0,6 & 12,3 & 3,1 & 20,0 & 12,0 & 6,6 & 7,1 & 600 \\
\hline 8 & SCC & 44 & M & moderada & 2 & 17,5 & 11,4 & 0,6 & 5,5 & 0,9 & 9,5 & 2,5 & 3,2 & 4,1 & 600 \\
\hline 9 & LHR & 22 & $M$ & moderada & 2 & 15,5 & 4,6 & 0,3 & 5,3 & 2,9 & 13,0 & 2,7 & 0,3 & 0,5 & 780 \\
\hline 10 & $\mathrm{BAB}$ & 28 & $\mathrm{~F}$ & intensa & 2 & 5,7 & 3,9 & 0,7 & 9,5 & 1,9 & 8,4 & 6,3 & 2,7 & 5,8 & 500 \\
\hline 11 & CSM & 55 & $M$ & moderada & 1 & 13,9 & 9,7 & 0,7 & 5,0 & 1,7 & 5,2 & 4,0 & 1,4 & 1,4 & 400 \\
\hline 12 & FOL & 53 & $\mathrm{M}$ & moderada & 1 & 1,2 & 2,1 & 1,7 & 1,6 & 1,0 & 12,0 & 4,7 & 6,8 & 3,2 & 450 \\
\hline 13 & FSL & 41 & $F$ & moderada & 1 & 5,5 & 3,8 & 0,7 & 4,5 & 0,7 & 4,5 & 1,2 & 1,2 & 0,8 & 470 \\
\hline 14 & NPS & 58 & $F$ & moderada & 1 & 4,7 & 3,2 & 0,7 & 3,2 & 0,8 & 4,5 & 1,2 & 1,5 & 2,1 & 600 \\
\hline 15 & MJA & 71 & $\mathrm{~F}$ & moderada & 1 & 1,1 & 1,3 & 1,2 & 3,9 & 0,7 & 9,6 & 4,4 & 0,8 & 2,0 & 400 \\
\hline 16 & LD & 66 & $\mathrm{M}$ & discreta & 2 & 3,8 & 1,5 & 0,4 & 3,1 & 0,3 & 4,4 & 3,2 & 0,7 & 1,7 & 600 \\
\hline 17 & AVS & 38 & M & moderada & 2 & 8,7 & 4,0 & 0,4 & 5,7 & 0,7 & 6,8 & 0,3 & 3,2 & 1,7 & 500 \\
\hline 18 & $\mathrm{PM}$ & 51 & M & moderada & 1 & 6,4 & 2,7 & 0,4 & 5,1 & 0,7 & 3,5 & 0,0 & 2,8 & 0,6 & 800 \\
\hline \multirow[t]{6}{*}{19} & ZSM & 57 & $\mathrm{~F}$ & moderada & 1 & 10,2 & 3,9 & 0,4 & 3,9 & 0,4 & 7,0 & 0,8 & 0,1 & 1,8 & 550 \\
\hline & & & & Média & & 10,9 & 6,1 & 0,6 & 5,9 & 1,3 & 7,5 & 2,9 & 2,2 & 2,3 & 543,1 \\
\hline & & & & DP & & 6,8 & 4,4 & 0,3 & 3,1 & 1,01 & 4,3 & 2,7 & 1,9 & 1,9 & 117,3 \\
\hline & & & & Mediana & & 10.2 & 4.0 & 0.6 & 5.1 & 0.9 & 6.8 & 2.7 & 1.5 & 1.8 & 500 \\
\hline & & & & Mínimo & & 1.1 & 1.3 & 0.3 & 1.6 & 0.3 & 2.0 & 0,0 & 0.1 & 0.3 & 400 \\
\hline & & & & Máximo & & 24.2 & 17.2 & 1.7 & 12.3 & 3.4 & 20.0 & 12.0 & 6.8 & 7.1 & 800 \\
\hline
\end{tabular}




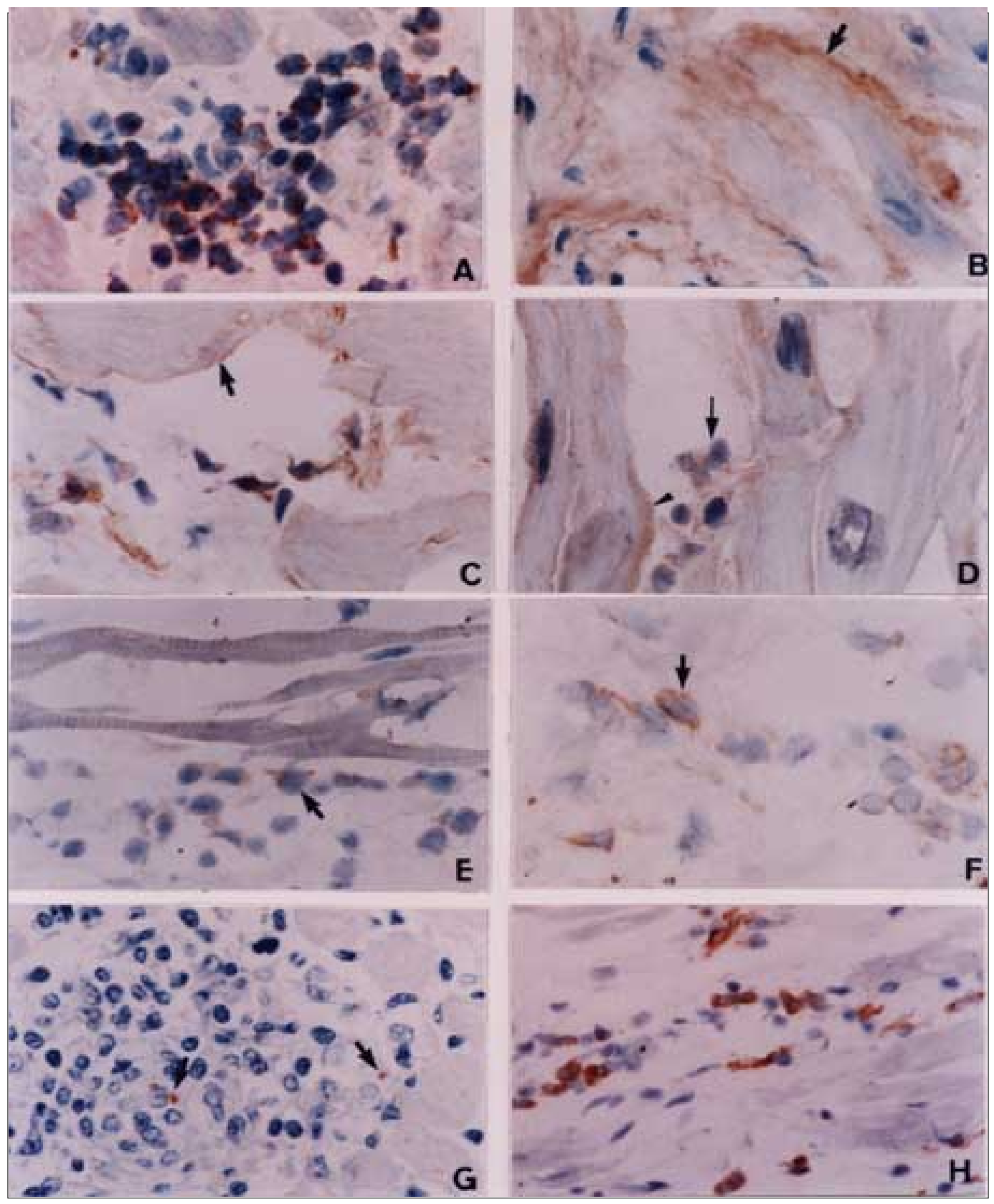

Figura 1 - Cortes histológiccos de miocárdios submetidos à coloração pela imunoperoxidase: (A) caso $n^{\circ} 17$, mostrando intensa marcação de células mononucleares para PDFG-A (X630); (B) o mesmo caso, apresentando positividade na matriz extracelular para PDGF-A (seta, x630); (C) intensa imunorreatividade em sarcolema (seta), células endoteliais e células intersticiais inflamatórias para PDGF-A no caso 12. Note-se que não há positividade no citoplasma das fibras cardíacas (x630): (D) caso $n^{\circ} 10$ mostrando marcacão em sarcolema (cabeça de seta) e células intersticiais inflamatórias (seta) para PDGF-B. Note-se que há discreta imunorreação no citoplasma das fibras cardíacas (x630): (E) caso $n^{\circ} 5$, mostrando poucas células mononucleares positivas para TGF- $\beta 1$, (seta, x630); $(F)$, caso $n^{\circ} 16$, mostrando foco de células inflamatórias GM-CSF positivas (seta, x630); (G) caso $n^{\circ}$ 8, mostrando foco inflamatório exibindo vários amastigotas na matriz extracelular, imunocorados com anti-T. cruzi (seta, x630): $(H)$, caso $n^{\circ} 5$, mostrando infiltrado inflamatório apresentado imunomarcação de células monucleares CD68+, correspondendo a macrófagos (x400). 
Todos os pacientes apresentaram miocardite com atividade discreta a intensa e a média do peso dos corações foi de $543,1 \mathrm{~g}( \pm 117,3)$, sendo que todos apresentavam peso aumentado, entre $400 \mathrm{~g}$ e $800 \mathrm{~g}$.

A Tabela 2 mostra a freqüência de positividade encontrada semiquantitativamente nas células endoteliais, células musculares lisas, sarcolema e matriz extracelular, agrupando-se os casos com intensidade 0 ou 1 e os de 2 ou 3 . Verificou-se que as porcentagens de casos com intensidade 2 ou 3 para o PDGF-A em células endoteliais, células musculares lisas e sarcolema (Figura 1C) foram de $63,1 \%, 57,9 \%$ e $52,6 \%$ respectivamente, ao passo que, na matriz extracelular, predominou $(63,1 \%)$ a intensidade 0 ou 1 (Figura 1B). Ao contrário, o PDGF-B apresentou maiores porcentagens com intensidade 0 ou 1 , sendo $94,7 \%$ para célula endotelial, $63,1 \%$ para célula muscular lisa e $89,5 \%$ para a matriz extracelular. No sarcolema houve discreto predomínio de intensidade 2 ou $3(52,6 \%)$.

O TGF- $\beta 1^{+}$foi encontrado apenas em sarcolema e as reações imunocoradas com este anticorpo foram sempre de intensidade cromogênica muito delicada, sugerindo baixa produção do mesmo (Figura 1E), embora tenhamos encontrado uma média de 2,2 de células intersticiais positivas para o TGF- $\beta 1$. Observamos que, para o TGF $\beta 1,100 \%$ dos casos apresentaram ausência ou discreta imunorreatividade em sarcolema (0 e 1), com média de 0,6 $( \pm 0,5)$.

Não encontramos positividade em células endoteliais e nem na matriz extracelular para o TGF- $\beta 1$ e para o GM-CSF, exceto o caso 19 , que apresentou discreta (intensidade 1) imunocoloração na matriz extracelular para o TGB- $\beta 1$. Quando a positividade para o PDGF-A e B no sarcolema era intensa, o citoplasma da fibra também estava bastante corado; quando era discreta a imunorreatividade no sarcolema o citoplasma da fibra ficava totalmente negativo (Figura 1C e 1D).

$O$ teste de correlação de Pearson mostrou forte correlação entre quantidade de células inflamatórias positivas para PDGF-A e PDGF-B $(r=0,73$ e $P=0,0004)$ e entre PDGF-B e GM-

Tabela 2 - Freqüência, média e desvio padrão da intensidade da semiquantificação das imuno-reações para PDGF-A, PDGF-B, TGF-b1 e GM-CSF em 19 pacientes com cardiopatia chagásica crônica.

\begin{tabular}{|c|c|c|c|c|c|c|c|c|c|}
\hline \multirow{2}{*}{$\begin{array}{l}\text { Fator de } \\
\text { crescimento }\end{array}$} & \multirow[t]{2}{*}{ Intensidade } & \multicolumn{2}{|c|}{$\begin{array}{c}\text { Célula } \\
\text { endotelial }\end{array}$} & \multicolumn{2}{|c|}{$\begin{array}{c}\text { Célula } \\
\text { muscular lisa }\end{array}$} & \multicolumn{2}{|c|}{ Sarcolema } & \multicolumn{2}{|c|}{$\begin{array}{c}\text { Matriz } \\
\text { extracelular }\end{array}$} \\
\hline & & $\mathrm{n}^{\circ}$ & $(\%)$ & $\mathrm{n}^{\circ}$ & $(\%)$ & $\mathrm{n}^{\circ}$ & $(\%)$ & $\mathrm{n}^{\circ}$ & $(\%)$ \\
\hline \multirow[t]{3}{*}{ PDGF-A } & 0 ou 1 & 7 & 36,8 & 8 & 42,1 & 9 & 47,3 & 12 & 63,1 \\
\hline & 2 ou 3 & 12 & 63,1 & 11 & 57,9 & 10 & 52,6 & 7 & 36,8 \\
\hline & Média (DP) & $1,89(1,0)$ & $2,0(0,9)$ & $1,78(0,9)$ & $1(1,1)$ & & & & \\
\hline \multirow[t]{3}{*}{ PDGF-B } & 0 ou 1 & 18 & 94,7 & 12 & 63,1 & 9 & 47,3 & 17 & 89,5 \\
\hline & 2 ou 3 & 1 & 5,2 & 7 & 36,8 & 10 & 52,6 & 2 & 10,5 \\
\hline & Média (DP) & $0,52(0,7)$ & $1,47(0,9)$ & $1,78(1,1)$ & $0,36(0,8)$ & & & & \\
\hline \multirow[t]{4}{*}{ TGF-b1 } & 0 ou 1 & 19 & 100 & 19 & 100 & 19 & 100 & 19 & 100 \\
\hline & 2 ou 3 & 0 & 0 & 0 & 0 & 0 & 0 & 0 & 0 \\
\hline & Média (DP) & 0 & 0 & $0,6(0,5)$ & 0 & & & & \\
\hline & 0 ou 1 & 19 & 100 & 19 & 100 & 19 & 100 & 19 & 100 \\
\hline \multirow[t]{2}{*}{ GM-CSF } & 2 ou 3 & 0 & 0 & 0 & 0 & 0 & 0 & 0 & 0 \\
\hline & Média (DP) & 0 & 0 & 0 & 0 & & & & \\
\hline
\end{tabular}

CSF $(r=0,67$ e $P=0,001)$. Não houve correlação estatisticamente significante entre PDGF-A e TGF- $\beta 1(r=0,59$ e $P=0,007)$, PDGF-A e GMCSF $(r=0,57$ e $P=0,009)$ nem entre PDGF-B e TGF- $\beta 1(r=0,56$ e $P=0,01)$. Também não houve correlação entre aumento de células inflamatórias intersticiais (CD8 ${ }^{+}, \mathrm{CD}^{+}, \mathrm{CD}^{2} 0^{+} \mathrm{e}$ $\mathrm{CD}^{+} 8^{+}$e aumento de células positivas para PDGF-A, PDGF-B, TGF- $\beta 1$ e GM-CSF, exceto quanto as células CD68+ e GM-CSF $(r=0,63$ e $\mathrm{P}=0,004)$.

O Teste de Wilcoxon mostrou não haver correlação entre número de células positivas para os fatores de crescimento e quantidade de antígenos do T. cruzi.

O coeficiente de correlação de Spearman mostrou não haver correlação entre quantidade 
de células intersticiais positivas para PDGF-A, PDGF-B eTGF- $\beta 1$ e positividade desses fatores em células endoteliais, células musculares lisas, matriz extracelular e no sarcolema da fibra cardíaca.

\section{DISCUSSÃO}

A fibrose é uma das graves complicações da CCC exercendo papel fundamental no desenvolvimento da insuficiência cardíaca crônica nesses pacientes. Fatores de crescimento podem estar envolvidos no desenvolvimento desse processo fibrótico em pacientes com doença de Chagas $^{39}$, sendo poucas as informações disponíveis a respeito, até o presente momento. Wyler et al ${ }^{39}$ demonstraram, em culturas de células de fibroblastos, musculares lisas e miocárdicas cultivadas com fator de crescimento para fibroblastos (FGF), que o parasitismo de células mesenquimais e de células miocárdicas resultava na elaboração de FGF e que, portanto, poderia ter papel na iniciação da fibrose miocárdica.

Trautmann et $\mathrm{al}^{37}$ demonstraram que a IL-4 derivada de mastócitos atuava como uma sinalização para produção de FGF e PDGF. Em trabalho recente, demonstramos a presença de IL-4 no infiltrado inflamatório do miocárdio de pacientes com $\mathrm{CCC}^{23}$, portanto, este mecanismo de indução de fatores de crescimento pode estar acontecendo nos chagásicos crônicos.

Silva et $\mathrm{al}^{31}$ observaram que macrofágos humanos tratados com o TGF- $\beta$, antes e depois da infecção das células, podem aumentar a replicação do T. cruzi no interior de macrófago, como bloquear os efeitos inibitórios da replicação do parasita induzido pelo IFN- $\gamma$ (interferongamma), sugerindo que o TGF- $\beta$ está associado com a regulação imune e com o controle da ativação de macrófagos em resposta a parasitas.

Em outras doenças, como nas renais, o TGF- $\beta 1$ regula a produção e renovação da matriz extracelular, como a fibronectina, proteoglicanos (decorina, biglicano) e colágeno do tipo IV ${ }^{5}$. É também quimiotático para fibroblastos e estimula o crescimento de fibroblastos imaturos. Assim, a produção do TGF- $\beta$ no local da lesão recruta macrófagos e fibroblastos que aumentam a produção in situ do TGF- $\beta$, juntamente com as células residentes ${ }^{22}$. Alguns autores têm sugerido que o TGF- $\beta$ poderia ter papel importante na resposta à lesão cardíaca. Isto foi demonstrado através de estudo imunohistoquímico onde células miocárdicas de ventrículo normal de rato ficaram fortemente positivas para TGF- $\beta$, enquanto que, durante o infarto do miocárdio causado pela ligadura de artéria coronariana, houve perda progressiva dessa coloração na fibra. Entretanto, 24 a 48 horas após a ligadura, encontrou-se intensa coloração dos miócitos na margem das áreas de infarto ${ }^{35}$. No presente trabalho, estudamos a CCC, onde há um processo de reparação provavelmente conseqüente a lesões isquêmicas ${ }^{12} 172036$. Apesar de ser esperado aumento do TGF- $\beta$, este fator esteve presente em baixa concentração, tendo-se encontrado grande quantidade de PDGF-A.

Não encontramos correlação entre presença de células $\mathrm{CD}^{+} 8^{+}$(macrófagos) e de células Te $B$ e presença de TGF- $\beta 1$ e, também, não houve correlação entre presença desse fator de crescimento e quantidade de antígenos de T. cruzi. Da análise quantitativa e semiquantitativa, verificamos que há baixa produção de TGF- $\beta 1$ na CCC e que, portanto, este não parece exercer um papel fundamental no desenvolvimento da fibrose nessa doença.

Além da apresentação de antígenos, os macrófagos também possuem muitas outras atividades funcionais, como a fagocitose de complexos imunes e atividades microbicidas e tumoricidas. O estado funcional dos macrófagos é regulado por várias substâncias, como endotoxinas e interferon- $\gamma^{1}$. Morrissey et $a^{18}$ observaram que o GM-CSF é capaz de promover aumento de receptor $\mathrm{Fc}$ em macrófagos peritoneais, aumentando a capacidade de apresentação de antígenos. No mesmo trabalho, os macrófagos peritoneais de camundongos infectados com T. cruzi quando incubados com GM-CSF, apresentaram menos parasitas por célula, e menos células infectadas, demonstrando que o GM-CSF melhorava a capacidade dos macrófagos em inibir a replicação do $T$. cruzir $^{18}$. No presente trabalho, analisando pacientes com CCC, não encontramos correlação entre quantidade de antígenos de T.cruzie quantidade de células inflamatórias imunomarcadas com anti- GM-CSF. Porém, assim como o TGF- $\beta 1$, a produção de GM-SCSF parece ser baixa nesses pacientes e, portanto, não deve exercer papel importante no desenvolvimento da fibrose. Por outro lado, esta baixa produção de GM-CSF pode ter papel patogênico auxiliar, favorecendo a permanência do parasita no hospedeiro.

O PDGF é um potente mediador de proliferação de células musculares lisas ${ }^{26}{ }^{32}$. O 
desenvolvimento de fibrose intersticial e de lesão vascular proliferativa são características da rejeição crônica renal. A forte expressão de fatores de crescimento, particularmente o PDGF e TGF- $\beta$ em endotélio e em células musculares lisas na rejeição crônica renal, sugere que eles poderiam ser os responsáveis pelo desenvolvimento das lesões vasculares. No presente estudo verificamos que, de todos esses fatores de crescimento estudados, houve predominância de PDGF-A e este fator esteve presente em células endoteliais, células musculares lisas e sarcolema; não houve, porém, correlação entre quantidade de células inflamatórias intersticiais positivas para o PDGF A e B e presença desses fatores nas várias localidades. Este achado indica que, provavelmente, a indução desses fatores não decorre apenas da inflamação e que a fibrose intersticial na doença de Chagas pode estar ligada à presença de PDGF mas não necessariamente à inflamação constante.

Em trabalho recente do nosso grupo, demonstrou-se que proteínas do complemento C5-b9 estão presentes no sarcolema das fibras cardíacas viáveis em pacientes com $\mathrm{CCC}^{2}$. A presença de proteínas do complemento C5-b9 (MAC - membrane attack complex of complement) na membrana de células, em quantidade subletal, constitui mecanismo indutor de liberação de fatores de crescimento, recentemente descrito. Isto provocaria, nessas células, a produção de FGF e PDGF ${ }^{4}$. Também em outras doenças, nas quais ocorre a proliferação celular, a presença de C5-b9 é uma das características que podem estar associadas à atividade proliferativa. Essas doenças seriam, por exemplo, a aterosclerose ${ }^{9}$, nefropatias ${ }^{19}$ e outras, onde o mecanismo patogênico seria semelhante-proliferação do tecido conjuntivo. A demonstração de MAC no sarcolema das fibras cardíacas viáveis nos pacientes chagásicos crônicos é um forte indicativo de que estas células podem estar sendo estimuladas a produzir fatores de crescimento. A liberação desses fatores de crescimento poderia explicar a fibrose fina envolvendo cada fibra miocárdica presente no coração de pacientes chagásicos crônicos. Corrobora esta idéia a observação de Wyler et al ${ }^{39}$, de que cultura de miócitos cardíacos, quando em presença de T. cruzi, apresenta presença de FGF no sobrenadante. Por outro lado, Speir et $\mathrm{al}^{33} 34$ observaram, em cultura de miócitos cardíacos não infectados, quantidade significante de FGF na fibra cardíaca de ratos adultos, quando quantificado pela técnica de radioimunoensaio.

A presença de MAC nas fibras cardíacas pode ser explicada pela indução de fenômenos imunológicos ativos contra o parasita, inclusive a presença de complemento. Parte desse complemento poderia se acumular nas fibras cardíacas, principalmente quando a resposta inflamatória contra o T. cruzi é exagerada. $O$ T. cruzi tem mecanismos de evasão à lise pelo complemento, pela produção de substâncias semelhantes aos fatores inibidores do complemento ${ }^{30} 40$.

Encontramos forte correlação entre números de células inflamatórias positivas para PDGF-A e PDGF-B e, também, entre PDGF-B e GM-CSF, além de correlação estatisticamente não significante entre PDGF-A e TGF- $\beta 1$, entre PDGF-A e GM-CSF e entre PDGF-B e TGF- $\beta 1$. Estes achados confirmam que, como citocinas, esses fatores de crescimento apresentam muitos efeitos, múltiplas interações e efeitos sinérgicos e antagonistas, complicando o entendimento de seus papéis biológicos na doença de Chagas.

Em conclusão, dos fatores de crescimento estudados, o PDGF (principalmente PDGF-A) possivelmente exerce papel fundamental na proliferação de células e, conseqüentemente, na produção da fibrose na CCC. Já os outros dois fatores estudados (GM-CSF e TGF- $\beta 1$ ) estão muito pouco expressos, sugerindo algum fator inibitório atuando sobre macrófagos, podendo corresponder à imunodepressão devido à presença de T. cruzi, que tem sido amplamente demonstrada.

\section{AGRADECIMENTOS}

Este trabalho teve auxílio da Fundação Euclides Jesus Zerbini, CNPq e FAPESP (processo no 1996/01075-7).

\section{REFERÊNCIAS BIBLIOGRÁFICAS}

1. Adams DO, Hamilton TA. Molecular transductional mechanisms by which IFN- $\gamma$ and other signals regulate macrophage development. Immunology Research 97:527, 1987.
2. Aiello VD, Reis MM, Benvenuti LA, Higuchi ML, Ramirez JAF. Expressão do Complexo Terminal do Complemento (C5b-9) e de um de seus inibidores (CD59) no miocárdio de pacientes com cardiopatia chagásica crônica $(\mathrm{CCH})$ 
e cardiopatia dilatada idiopática (CMD). Arquivos Brasileiros de Cardiologia 73 (supl IV):65, 1999.

3. Ben Younès-Chennoufi $A$, Hontebeyrie-Joskowicz $M$, Tricottet V, Eisen H, Reynes M, Said G. Persistence of Trypanosoma cruzi antigens in the inflammatory lesions of chronically infected mice. Transactions of the Royal Society of Tropical Medicine and Hygiene 82:77-83, 1988.

4. Benzaquen LR, Nicholson-Weller A, Halperin JA. Terminal complement proteins $\mathrm{C} 5 \mathrm{~b}-9$ release basic fibroblast growth factor and platelet-derived growth factor from endothelial cells. Journal Experimental of Medicine 179:985-992, 1994

5. Border WA, Okuda S, Languino LR, Ruoslahti E. Transforming growth factor $b$ regulates production of proteoglycans by mesangial cells. Kidney International 37:689-695, 1990.

6. Chagas C. Processos patojenicos da tripanozomiase americana. Memórias do Instituto Oswaldo Cruz 8:5-36, 1916.

7. Cossio PM, Diez C, Szarfman A, Kreutzer E, Candiolo B, Arana RM. Chagasic cardiopathy. Demonstration of a serum gamma factor globulin factor which reacts with endocardium and vascular structures. Circulation 49:1321, 1974.

8. Cunha Neto E, Rizzo LV, Albuquerque F, Abel L, Guilherme L, Bocchi E, Bacal F, Carrara D, Ianni B, Mady C, Kalil J. Cytokine production profile of heartinfiltrating T cells in Chagas' disease cardiomyopathy. Brazilian Journal of Medicine and Biology Research 31:133-137, 1998.

9. Hachida M, Zhang X, Lu H, Hoshi H, Furutani Y, Matsuoka $\mathrm{R}$, Koyanagi $\mathrm{H}$. Association between the degree of platelet-derived growth factor-A chain mRNA expression and coronary arteriosclerosis in the transplanted heart. Heart Vessels 13:24-29, 1998.

10. Heppleston AG, Styles JA. Activity of a macrophage factor in collagen formation by silica. Nature 214: 521-522, 1967.

11. Higuchi ML, Brito T, Reis MM, Barbosa A, Bellotti G, Pereira-Barreto AC, Pileggi F. Correlation between Trypanosoma cruzi parasitism and myocardial inflammatory infiltrate in human chronic chagasic myocarditis: light microscopy and immunohistochemical findings. Cardiovascular Pathology 2:101-106, 1993.

12. Higuchi ML, Fukasawa S, Brito T, Parzianello LC, Bellotti G, Ramirez JA. Different microcirculatory and interstitial matrix patterns in idiopathic dilated cardiomyopathy and Chagas' disease: a three dimensional confocal microscopy study. Heart 82:279-285, 1999.

13. Hirabayashi T, Demertzis S, Schafers J, Hoshino K, Nashan B. Chronic rejection in lung allografts: immunohistological analysis of fibrogenesis. Transplantation International 9 (suppl 1):S 293-295, 1996.

14. Jones EM, Colley DG, Tostes Jr S, Lopes ER, Vnencak Jones CL, McCurley TL. Amplification of Trypanosoma cruzi DNA sequence from human inflammatory lesions in human chagasic cardiomyopathy. American Journal of Tropical Medicine and Hygiene 48:348-357, 1993.

15. Kehrl JH, Wakefield LM, Roberts AB, Jakowlew S, Alvarez-Mon M, Derynck R, Sporn MB, Fauci AS. Production of transforming growth factor beta by human $T$ lymphocytes and its potential role in the regulation of $T$ cell growth. Journal of Experimental Medicine 163:10371050, 1986.

16. Leibovich SJ, Ross R. A macrophage-dependent factor that stimulates the proliferation of fibroblasts in vitro. American Journal of Pathology 84:501-514, 1976.

17. Morais CF, Higuchiml, Lage S. Chagas' heart disease and myocardial infarct. Incidence and report of four necropsy cases. Annals of Tropical Medicine and Parasitology 83:207-214, 1989.

18. Morrissey PJ, Grabstein KH, Reed SG, Colon PJ. Granulocyte/macrophage colony stimulating factor. A potent activation signal for mature macrophages and monocytes. International Archives of Allergy and Applied Immunology 88:40-45, 1989.

19. Noronha IL, Niemir Z, Stein H, Waldherr R. Cytokines and growth factors in renal disease. Nephrology, Dialysis, Transplantation 10: 775-786, 1995.

20. Oliveira JSM, Araujo RRC, Navarro MA, Muccillo G. Cardiac thrombosis and thromboembolism in chronic Chagas' heart disease. American Journal of Cardiology 52:147-151, 1983.

21. Pereira-Barreto AC, Mady C, Arteaga-Fernandes E, Stolf N, Lopes EA, Higuchiml, Bellotti G, Pileggi F. Right ventricular endomyocardial biopsy in chronic Chagas' disease. American Heart Journal 111:307-312, 1986.

22. Postleythwaite AE, Keski-Oja J, Moses HL, Kang AH. Stimulation of the chemotactic migration of the human fibroblast by transforming growth factor $b$. Journal Experimental of Medicine 165:251-256,1987.

23. Reis MM, Higuchiml, Benvenuti LA, Aiello VD, Gutierrez PS, Belotti G, Pileggi F. An in situ quantitative immunohistochemical study of cytokines and IL-2R+ in chronic human chagasic myocarditis: correlation with the presence of myocardial Trypanosoma cruzi antigens. Clinical Immunology and Immunopathology 83:165-172, 1997.

24. Rosner B. Nonparametric methods. In: Fundamentals of Biostatistics, $2^{\text {nd }}$ edition, PWS Publishers, Massachusetts. p. 288-293, 1986.

25. Rosner B. Regression and correlation methods. In: Fundamentals of Biostatistics, $2^{\text {nd }}$ edition, PWS Publishers, Massachusetts. p. 419-422, 1986.

26. Ross R, Glomset J, Kariya B, Harker L. A plateletdependent serum factor that stimulates the proliferation of arterial smooth muscle cells in vitro. Proceeding of the National Academy of Sciences of the United States of American 71:1207-1210, 1974 
27. Santos-Buch CA, Teixeira ARL. Immunology of experimental Chagas' disease III. Rejection of allogeneic heart cells in vitro. Journal of Experimental Medicine 140:398-402, 1988.

28. Statistical Analysis Software/STAT" User's Guide, version 6, $4^{\text {th }}$ edition, SAS Institute Inc., Cary, NC, v. 1, 1989.

29. Statistical Analysis Software/STAT" User's Guide, version 6, $4^{\text {th }}$ edition, SAS Institute Inc., Cary, NC, v. 2, 1989.

30. Schonermark S, Rauterberg EW, Shinml, Loke S, Roelcke D, Hansch GM. Homologous species restriction in lysis of human erythrocytes: a membrane-derived protein with C8-binding capacity functions as an inhibitor. Journal of Immunology 136:1772-1776, 1986.

31. Silva JS, Twardzik DR, Reed SG. Regulation of Trypanosoma cruzi infections in vitro and in vivo by transforming growth factor $b(T G F-\beta)$. Journal of Experimental Medicine 174:539-545, 1991.

32. Sjolund M, Hedin U, Sejersen T, Heldin CH, Thyberg J. Arterial smooth muscle cells express platelet-derived growth factor (PDGF) A chain mRNA, secrete a PDGFlike mitogen, and bind exogenous PDGF in a phenotypeand growth state-dependent manner. Journal of Cell Biology 106:403-413 1988.

33. Speir E, Tanner V, Gonzales AM, Farris J, Baird A, Casscels W. Acidic and basic fibroblast growth factors in adult rat heart myocytes. Localization, regulation in culture, and effects on DNA synthesis. Circulation Research 71:251-259, 1992.

34. Speir E, Zhou YF, Lee M, Shrivastav S, Casscels W. Fibroblast growth factors are present in adult cardiac myocytes in vivo. Biochemical and Biophysical Research Communication 157: 1336-1340, 1989.

35. Thompson NL, Bazoberry F, Speir EH, Casscells W, Ferrans VJ, Flanders KC, Kondaiah P, Geiser AG, Sporn MB. Transforming growth factor beta-1 in acute myocardial infarction in rats. Growth Factors 1:91-99, 1988.

36. Torres CM. Sobre a anatomia patológica da doença de Chagas. Memórias do Instituto Oswaldo Cruz 36:391404, 1941.

37. Trautmann A, Krohne G, Brocker ER, Klein CE. Human mast cells augment fibroblast proliferation by heterotypic cell-cell adhesion and action of IL-4. Journal of Immunology 160:5053-5057, 1998.

38. Vianna G. Contribuição para o estudo da anatomia patolójica da moléstia de Carlos Chagas. (Esquizotripanoze humana ou tireoidite parazitária). Memórias do Instituto Oswaldo Cruz 3:276-294, 1911.

39. Wyler DJ, Libby P, Prakash S, Prioli RP, Pereira ME. Elaboration by mammalian mesenchymal cells infected with Trypanosoma cruzi of a fibroblast-stimulating factor that may contribute to chagasic cardiomyopathy. Infection and Immunity 55:3188-3191, 1987.

40. Zalman LS, Wood LM, Muller-Elberhard HJ. Isolation of a human erythrocyte membrane protein capable of inhibiting expression of homologous complement transmembrane channels. Procceeding of the National Academy of Sciences of the United States of America 83: 6975-6979, 1986. 